\title{
The predicted $\sigma^{54}$-dependent regulator EtpR is essential for expression of genes for anaerobic $p$-ethylphenol and $p$-hydroxyacetophenone degradation in "Aromatoleum aromaticum" EbN1
}

Imke Büsing ${ }^{1}$, Mirjam Kant ${ }^{1}$, Marvin Dörries ${ }^{1}$, Lars Wöhlbrand ${ }^{1}$ and Ralf Rabus ${ }^{1,2^{*}}$

\begin{abstract}
Background: The denitrifying betaproteobacterium "Aromatoleum aromaticum" EbN1 anaerobically utilizes a multitude of aromatic compounds via specific peripheral degradation routes. Compound-specific formation of these catabolic modules is assumed to be mediated by specific transcriptional activators. In case of the recently elucidated $p$-ethylphenol/p-hydroxyacetophenone pathway, the highly substrate-specific regulation was implicated to involve the predicted $\sigma^{54}$-dependent, NtrC-type regulator EbA324. The latter was suggested to control the expression of the two neighboring gene clusters encoding the catabolic enzymes as well as a corresponding putative solvent efflux system. In the present study, a molecular genetic approach was used to study the predicted function of EbA324.
\end{abstract}

Results: An unmarked in frame $\triangle e b A 324$ (here renamed as $\triangle e t p R ;$-ethylphenol regulator) deletion mutation was generated. The $\Delta$ etpR mutant was unable to grow anaerobically with either $p$-ethylphenol or $p$-hydroxyacetophenone. Growth similar to the wild type was restored in the $\Delta e t p R$ mutant background by in trans expression of plasmid-born etpR. Furthermore, expression of the "p-ethylphenol" gene clusters as well as corresponding protein formation was shown to depend on the presence of both, EtpR and either $p$-ethylphenol or $p$-hydroxyacetophenone. In the wild type, the etpR gene appears to be constitutively expressed and its expression level not to be modulated upon effector presence. Comparison with the regulatory domains of known phenol- and alkylbenzene-responsive NtrC-type regulators of Pseudomonas spp. and Thauera aromatica allowed identifying $>60$ amino acid residues in the regulatory domain (in particular positions 149 to 192 of EtpR) that may contribute to the effector specificity viz. presumptively restricted effector spectrum of EtpR.

Conclusions: This study provides experimental evidence for the genome predicted $\sigma^{54}$-dependent regulator EtpR (formerly EbA324) of "A. aromaticum" EbN1 to be responsive to $p$-ethylphenol, as well as its degradation intermediate $p$-hydroxyacetophenone, and to control the expression of genes involved in the anaerobic degradation of these two aromatic growth substrates. Overall, the presented results advance our understanding on the regulation of anaerobic aromatic compound catabolism, foremost based on the sensory discrimination of structurally similar substrates.

Keywords: $\sigma^{54}$-Dependent regulator, Aromatic compounds, $p$-Ethylphenol, $p$-Hydroxyacetophenone, Anaerobic degradation, "Aromatoleum aromaticum" EbN1, In frame deletion mutation, Proteomics, Gene expression, Sequence comparison

\footnotetext{
* Correspondence: rabus@icbm.de

${ }^{1}$ Institute for Chemistry and Biology of the Marine Environment (ICBM), Carl

von Ossietzky University Oldenburg, Oldenburg, Germany

${ }^{2}$ Max Planck Institute for Marine Microbiology, Bremen, Germany
} 


\section{Background}

The "Aromatoleum"/Thauera/Azoarcus cluster within Betaproteobacteria comprises most of the currently known denitrifiers capable of anaerobic degradation of aromatic compounds [1, 2]. "Aromatoleum aromaticum" EbN1 is a metabolically versatile and the first genomesequenced representative of this cluster, completely oxidizing $>20$ different aromatic compounds under anoxic conditions. These include the alkylbenzenes toluene and ethylbenzene, phenol, as well as the alkylphenols $p$-cresol and $p$-ethylphenol [3-5]. The multiple sensory/regulatory proteins predicted from the genome were suggested to constitute a fine-tuned regulatory network [6]. Subsequent experimental studies indeed implicated the latter in substratespecific formation of catabolic modules $[4,5,7,8]$, as well as in the adaptation to substrate-limiting [9] and stress conditions [10]. Synthesis of the hitherto accomplished physiological-proteomic insights qualifies "A. aromaticum" EbN1 as a promising systems biology model [11].

The anaerobic degradation of the growth substrates $p$-ethylphenol and $p$-hydroxyacetophenone by "A. aromaticum" EbN1 was recently shown to be analogous to the ethylbenzene pathway [5]. Initial oxygenindependent hydroxylation to 1-(4-hydroxyphenyl)-ethanol and subsequent dehydrogenation to $p$-hydroxyacetophenone is followed by decarboxylation and thiolytic cleavage yielding $p$-hydroxybenzoyl-CoA. Proteins involved in this degradation pathway and a presumptively associated efflux system are encoded in a $15 \mathrm{kbp}$ "catabolic" and a $6.5 \mathrm{kbp}$ "efflux" gene cluster located in direct neighborhood on the chromosome of "A. aromaticum" EbN1. The specific transcriptional induction of these two "p-ethylphenol" gene clusters in the presence of $p$-ethylphenol as well as $p$ hydroxyacetophenone was previously inferred from the highly similar abundance profiles of respective transcripts and proteins [5]. This substrate-specific regulation was suggested to be mediated by the predicted $\sigma^{54}$-dependent regulator EbA324 (here renamed as EtpR for p-ethylphenol regulator) that is encoded in between the "catabolic" and "efflux" gene clusters (Fig. 1a). Typically, $\sigma^{54}$-dependent regulators consist of (i) an $\mathrm{N}$-terminal regulatory, (ii) a central ATP-hydrolyzing (activating) and (iii) a C-terminal DNAbinding domain $[12,13]$. Upon effector-binding, such regulators oligomerize and bind to distinct DNA-enhancer sequences upstream of the transcriptional start site [14]. In the process of transcription initiation, $\sigma^{54}$ binds to a highly conserved $-12 /-24$ consensus sequence (5'-TGGC- $\mathrm{N}_{7^{-}}$ TTGCA-3') and recruits RNA polymerase and the $\sigma^{54}$ dependent regulator; the now formed RNA polymerase holoenzyme is activated by ATP-hydrolysis at the central domain of the regulator [15]. In accord, the promotor regions of the p-ethylphenol-related "catabolic" and "efflux" gene clusters in " $A$. aromaticum" EbN1 contain conserved $\sigma^{54}$-DNA-binding motifs [5]. Regulation of aerobic aromatic compound degradation by $\sigma^{54}$-dependent regulators has been well-studied in Pseudomonas spp. The NtrC-type regulators XylR and DmpR induce transcription of gene clusters for aerobic toluene/xylene [16] and phenolic compound degradation [17], respectively. In both cases, direct binding of the aromatic substrates or structurally related compounds to the regulatory domain relieves the repression of the ATPase activity of the central domain, allowing ATP-hydrolysis and subsequent transcription initiation $[18,19]$.

In the present study, an unmarked $\triangle \operatorname{etpR}$ in frame deletion mutation was generated to verify the predicted regulatory function of EtpR. The $\Delta e t p R$ mutant and the in trans etpR-complemented mutant were characterized by means of physiological experiments as well as on the molecular level.

\section{Results and discussion}

Generation of the $\Delta e t p R$ and etpR-complemented mutants The unmarked in frame $\Delta e t p R$ deletion mutation was generated to test the predicted regulatory function of EtpR in mediating $p$-ethylphenol- and $p$-hydroxyacetophenonespecific expression of the two " $p$-ethylphenol" gene clusters. In the $\triangle e t p R$ mutant, only the start and stop codons of $\operatorname{etp} R$ were preserved to maintain the reading frame (Fig. 1a). Accordingly, no PCR product could be obtained using etpR-specific primers and only a small 318 bp amplicon was observed applying primers targeting the up- and downstream intergenic regions of etpR (wild type amplicon 2,133 bp; Fig. 1b). In addition, correctness of the deletion site and its 5'- and 3'-flanking regions (i.e., $\Delta e t p R$ genotype) was confirmed by nucleotide sequencing (Additional file 1: Figure S1). This newly generated $\Delta e t p R$ mutant was in trans complemented via an etpR-bearing broad-host range plasmid, yielding constitutive expression of etpR (see below). The resultant etpR-complemented mutant had the genotype $\triangle e t p R$, pBBR1MCS-2 $\Omega e t p R$.

\section{The $\Delta$ etpR mutant cannot grow with $p$-ethylphenol and $p$-hydroxyacetophenone}

Detailed growth analyses of the $\triangle e t p R$ mutant were performed in comparison to the wild type and the etpR-complemented mutant. All three strains were benzoate-adapted and transferred at $1 / 2 \mathrm{OD}_{\max }$ to fresh media containing either benzoate, $p$-ethylphenol, $p$-hydroxyacetophenone or a binary mixture of benzoate and $p$-hydroxyacetophenone as sole source(s) of carbon and energy. Selected growth parameters are compiled in Table 1.

\section{Growth with benzoate}

Growth of the wild type and the $\Delta \operatorname{etpR}$ mutant with benzoate was nearly identical, while the etpR-complemented mutant grew slightly faster, despite the presence of kanamycin in the medium (Fig. 2b). Furthermore, the $\triangle e t p R$ mutant displayed growth similar to the wild type 


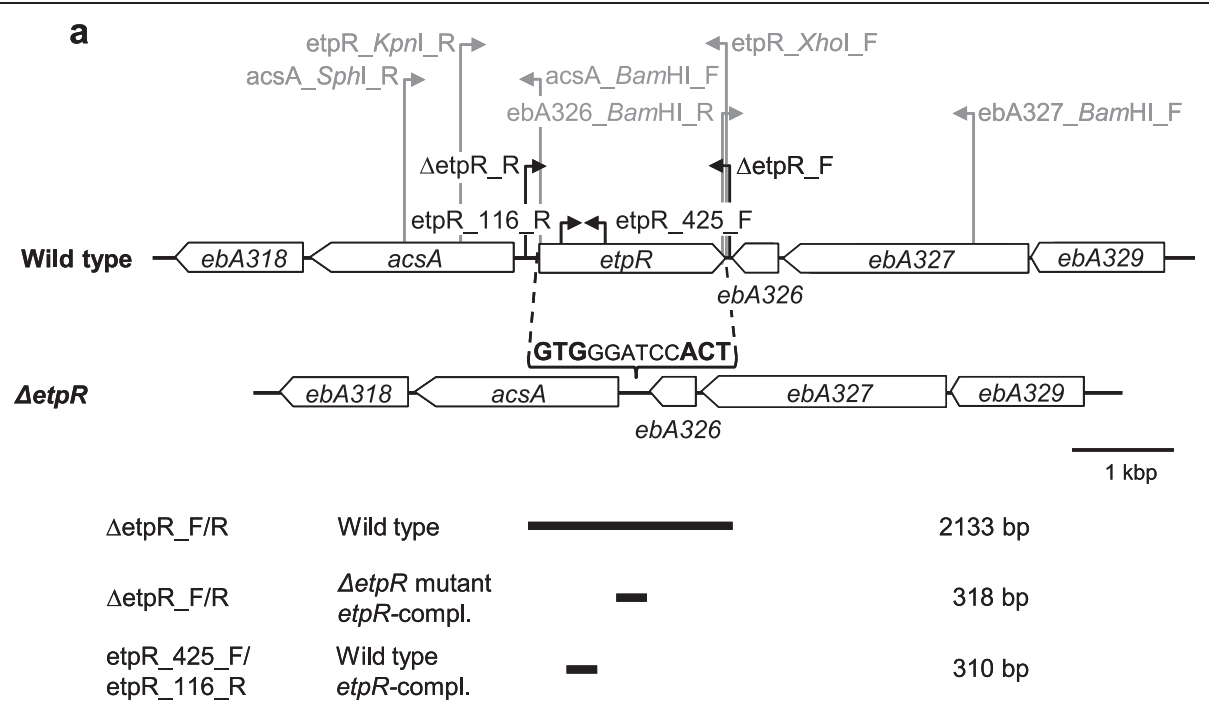

b

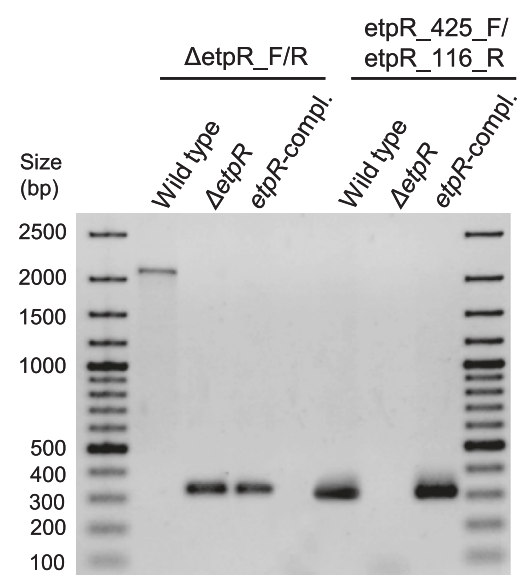

Fig. 1 Generation of $\Delta$ etpR deletion and etpR-complemented mutants. Scale model of enlarged section of the "p-ethylphenol" gene clusters on the chromosome of "A. aromaticum" EbN1, displaying etpR and its 3'- and 5'-neighbouring regions in the wild type (top) and the $\Delta e t p R$ mutant

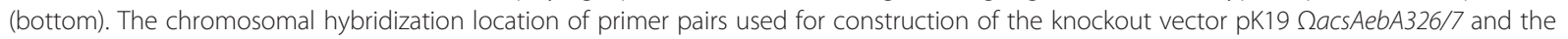
complementation plasmid pBBR1MCS-2 SetpR are shown in grey (Tables 2 and 3). The positions of the primer pair $\Delta$ etpR_F/R for identifying the knockout genotype and an etpR-specific primer pair are indicated in black and the lengths of the corresponding PCR products are given below the gene model (a). Electropherogram of PCR products obtained from the wild type, the $\Delta$ etpR mutant and the etpR-complemented mutant applying different primer pairs. Using the primer pair $\Delta e t p R \_F / R$, a 2,133 bp long amplicon was obtained for the chromosome of the wild type and a shorter, $318 \mathrm{bp}$ long, amplicon for the $\Delta e t p R$ mutant and the etpR-complemented mutant. Accordingly, amplification of etpR (310 bp) was not possible for the $\Delta e t p R$ mutant, but for the etpR-complemented mutant (b). Abbreviations: $\Delta$ etpR, $\Delta \operatorname{etp} R$ mutant; etpR-compl., etpR-complemented mutant

with all other known substrates (data not shown), except for $p$-ethylphenol and $p$-hydroxyacetophenone.

\section{Growth with p-ethylphenol and p-hydroxyacetophenone}

The $\Delta$ etpR mutant could not grow with $p$-ethylphenol and $p$-hydroxyacetophenone (Fig. 2a, c). The minute initial increase in optical density can probably be attributed to consumption of residual benzoate, co-transferred during initial inoculation. The unexpected complete depletion of $p$-ethylphenol from $\triangle e t p R$ mutant cultures after $\sim 160 \mathrm{~h}$ of incubation is most likely due to unspecific membrane accumulation, as it is not coupled to denitrification and also observable in wild type cultures with de novo protein synthesis inhibited by kanamycin (see Additional file 1: Figure S2). Noteworthy, the etpRcomplemented mutant started to grow after a markedly shorter lag-phase as compared to the wild type, even though maximum growth rates and substrate depletion profiles of the two strains were similar (Fig. 2a, c; Table 1). This shorter lag-phase may be due to the higher EtpR abundance in the complemented strain (see below). 
Table 1 Selected growth parameters determined for wild type, $\Delta$ etpR mutant and etpR-complemented mutant (etpR-compl.) of "A. aromaticum" EbN1 ${ }^{\text {a }}$

\begin{tabular}{|c|c|c|c|c|c|}
\hline \multirow[b]{2}{*}{ Substrate $(s)^{b}$} & \multirow[b]{2}{*}{ Genotype $^{d}$} & \multirow[b]{2}{*}{$\mu_{\max }\left(h^{-1}\right)$} & \multicolumn{2}{|c|}{ Consumption rate $\left(\mathrm{mM} \mathrm{h}^{-1}\right)$} & \multirow[b]{2}{*}{ Lag-phase (h) } \\
\hline & & & Substrate 1 & Substrate 2 & \\
\hline & wild type & 0.121 & 0.35 & - & 10 \\
\hline \multirow[t]{3}{*}{ Bz } & $\Delta e t p R$ & 0.121 & 0,34 & - & 10 \\
\hline & etpR-compl. & 0.130 & 0.40 & - & 9 \\
\hline & wild type & 0.064 & 0.03 & - & 57 \\
\hline \multirow[t]{3}{*}{ pHac } & $\Delta e t p R$ & - & - & - & - \\
\hline & etpR-compl. & 0.067 & 0.05 & - & 28 \\
\hline & wild type & 0.093 & 0.07 & - & 29 \\
\hline \multirow[t]{3}{*}{ pEp } & $\Delta e t p R$ & - & 0.01 & - & - \\
\hline & etpR-compl. & 0.098 & 0.08 & - & 20 \\
\hline & wild type & 0.067 & 0.11 & 0.03 & 13 \\
\hline \multirow[t]{3}{*}{$\mathrm{Bz}^{1}+\mathrm{pHac}^{2 \mathrm{c}}$} & $\Delta e t p R$ & 0.082 & 0.14 & - & 13 \\
\hline & etpR-compl. & 0.053 & 0.06 & 0.05 & 15 \\
\hline & wild type & 0.091 & 0.14 & 0.08 & 14 \\
\hline \multirow[t]{2}{*}{$B z^{1}+p E p^{2 c}$} & $\Delta e t p R$ & 0.096 & 0.14 & 0.05 & 14 \\
\hline & etpR-compl. & 0.135 & 0.12 & 0.08 & 16 \\
\hline
\end{tabular}

${ }^{a}$ All growth experiments were performed in duplicates with high reproducibility; the given values are mean values

${ }^{\mathrm{b}} \mathrm{Bz}$, benzoate; pHac, $p$-hydroxyacetophenone; $\mathrm{pEp}$, $p$-ethylphenol

"Superscript "1" denotes substrate 1; superscript " 2 " denotes substrate 2

${ }^{\mathrm{d}} \Delta$ etpR, $\triangle$ etpR mutant; etpR-compl., etpR-complemented mutant

\section{Growth with a binary mixture of benzoate and p-hydroxyacetophenone}

When the three strains were shifted from benzoate to a binary mixture of benzoate and $p$-hydroxyacetophenone, highest rates of growth and benzoate consumption were observed for the $\triangle e t p R$ mutant (Table 1), while the concentration of $p$-hydroxyacetophenone remained unchanged in this strain (Fig. 2d). However, growth rate and $\mathrm{OD}_{\max }$ were lower as compared to growth with benzoate, most likely due to the toxicity of $p$-hydroxyacetophenone. Rates for growth and benzoate consumption of the wild type with the binary substrate mixture were higher as compared to the etpR-complemented mutant, but the rate of concomitant $p$-hydroxyacetophenone depletion was lower (Table 1). Hence, the etpR-complemented mutant seems to more preferentially consume $p$-hydroxyacetophenone than benzoate as compared to the wild type (Fig. 2d). Similar results were obtained for growth experiments with a binary mixture of benzoate and $p$-ethylphenol (Additional file 1: Figure S3).

\section{EtpR mediates substrate-specific expression of the "p-ethylphenol" gene clusters}

The simultaneous utilization of benzoate and $p$-hydroxyacetophenone (see preceeding paragraph), i.e., absence of catabolite repression, is a prerequisite for subsequent analysis of transcript and proteomic profiles. In the $\triangle e t p R$ mutant benzoate sustains growth while at the same time the hypothesized loss of response to the effector $p$-hydroxyacetophenone due to etpR deletion can be tested. $p$-Hydroxyacetophenone was used as substitute of $p$-ethylphenol due to (i) its higher water solubility (not requiring provision via a carrier phase), (ii) the apparent absence of a passive uptake as observed for $p$-ethylphenol (Fig. 2a, c) and (iii) the uniform induction of gene expression and protein formation by both substrates [5].

Gene expression was analyzed for the wild type, the $\triangle e t p R$ mutant and the etpR-complemented mutant anaerobically grown with benzoate as single substrate or a binary mixture of benzoate and $p$-hydroxyacetophenone. As reference, transcripts were analyzed for $p$-hydroxyacetophenone-grown cells of the wild type and the etpRcomplemented mutant. Target genes were located at the beginning, in the middle and at the end of the two "p-ethylphenol" gene clusters, i.e., acs $A$, hped and $p c h F$ for the "catabolic" gene cluster and $e b A 335, e b A 327$ and $e b A 326$ for the "efflux" gene cluster (Fig. 3a). Transcripts of both gene clusters were only detected in wild type and etpR-complemented mutant cells growing with either $p$ hydroxyacetophenone or the binary substrate mixture 


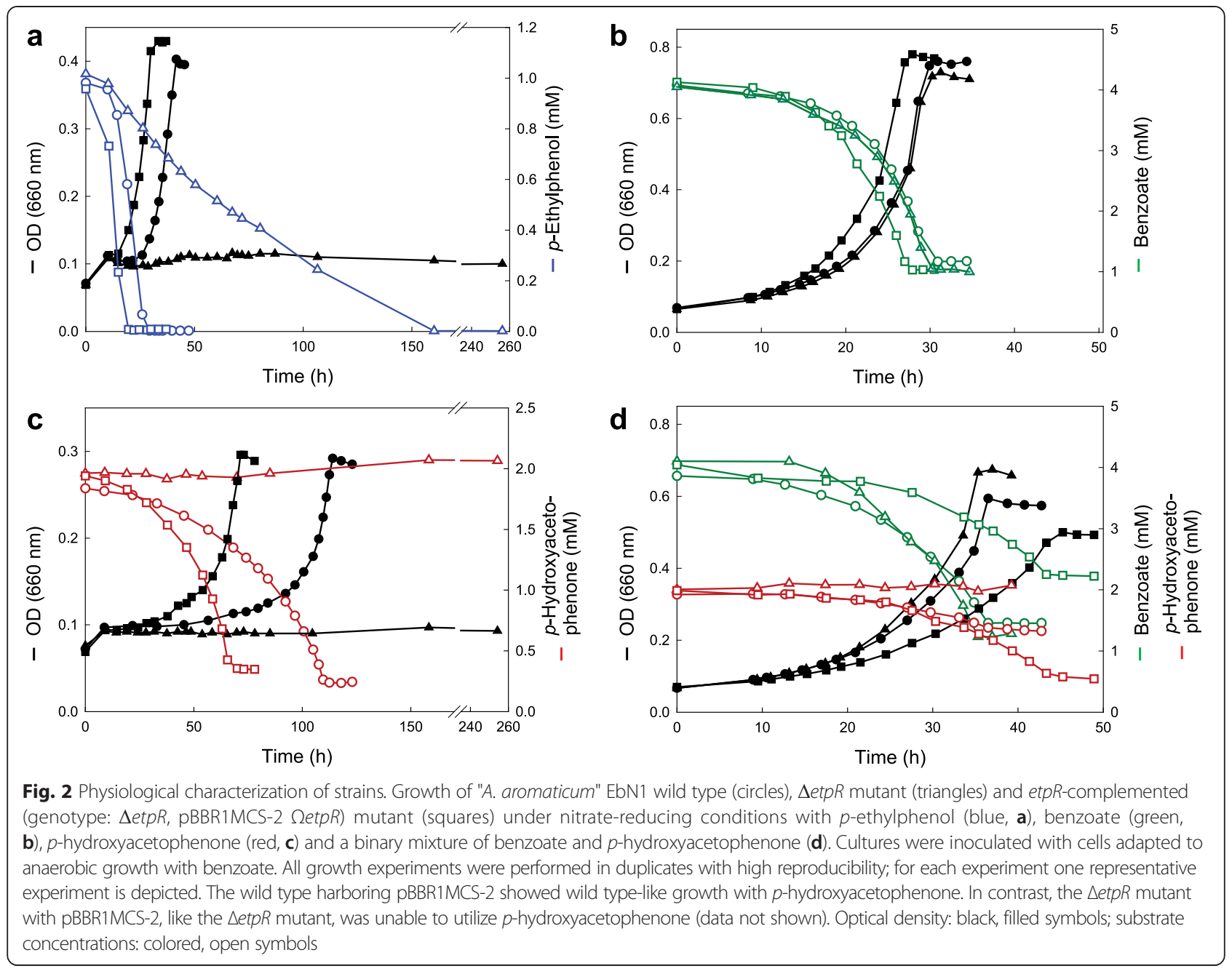

(Fig. 3b). In accord, 16 out of the 24 proteins encoded in the two "p-ethylphenol" gene clusters were only detected in the corresponding subproteoms. In case of the $\Delta e t p R$ mutant, none of these transcripts or proteins was detectable in cells grown with the binary substrate mixture (Fig. 3b). Similar proteomic results were obtained with a binary mixture of benzoate and $p$-ethylphenol (Additional file 1: Figure S3). Hence, expression of the " $p$-ethylphenol" gene clusters depends on the presence of EtpR and either $p$ ethylphenol or $p$-hydroxyacetophenone. The expression of genes framing the knockout locus (i.e., $e b A 327, e b A 326$ and acs $A$ ) by the etpR-complemented mutant in the presence of $p$-hydroxyacetophenone confirms the absence of polar effects arising from in frame deletion of the etpR gene.

Non-detection of the EtpR protein in the wild type suggests protein abundance below the detection limit of the applied method. In contrast, EtpR was detected in all samples of the etpR-complemented mutant with high confidence indicating a markedly higher abundance of EtpR. The absence of "p-ethylphenol" gene cluster expression in benzoate-utilizing cells of the etpR- complemented mutant, despite the artificially high EtpR abundance in this strain, demonstrates the effector dependence of EtpR for transcriptional activation.

\section{Hints on constitutive expression of etpR}

Binding of the XylR protein to the upstream region of the $x y l R$ gene was previously reported to reduce the $\sigma^{70}$ dependent expression of the latter, by what XylR negatively influences its own formation; moreover, effector presence was shown to enhance this effect $[16,20,21]$. To investigate if a similar feedback mechanism exists for EtpR, transcription of etpR was quantitatively analyzed in the wild type and the etpR-complemented mutant grown with benzoate, $p$-hydroxyacetophenone and a binary mixture of benzoate and $p$-hydroxyacetophenone. The wild type displayed even expression levels (-1.2-fold; Fig. 4) independent of the growth substrate(s) and effector presence, respectively. If EtpR influenced its own formation as reported for XylR, i.e. stronger decrease of expression due to effector presence, a significantly higher etpR expression level would have to be expected for the wild 


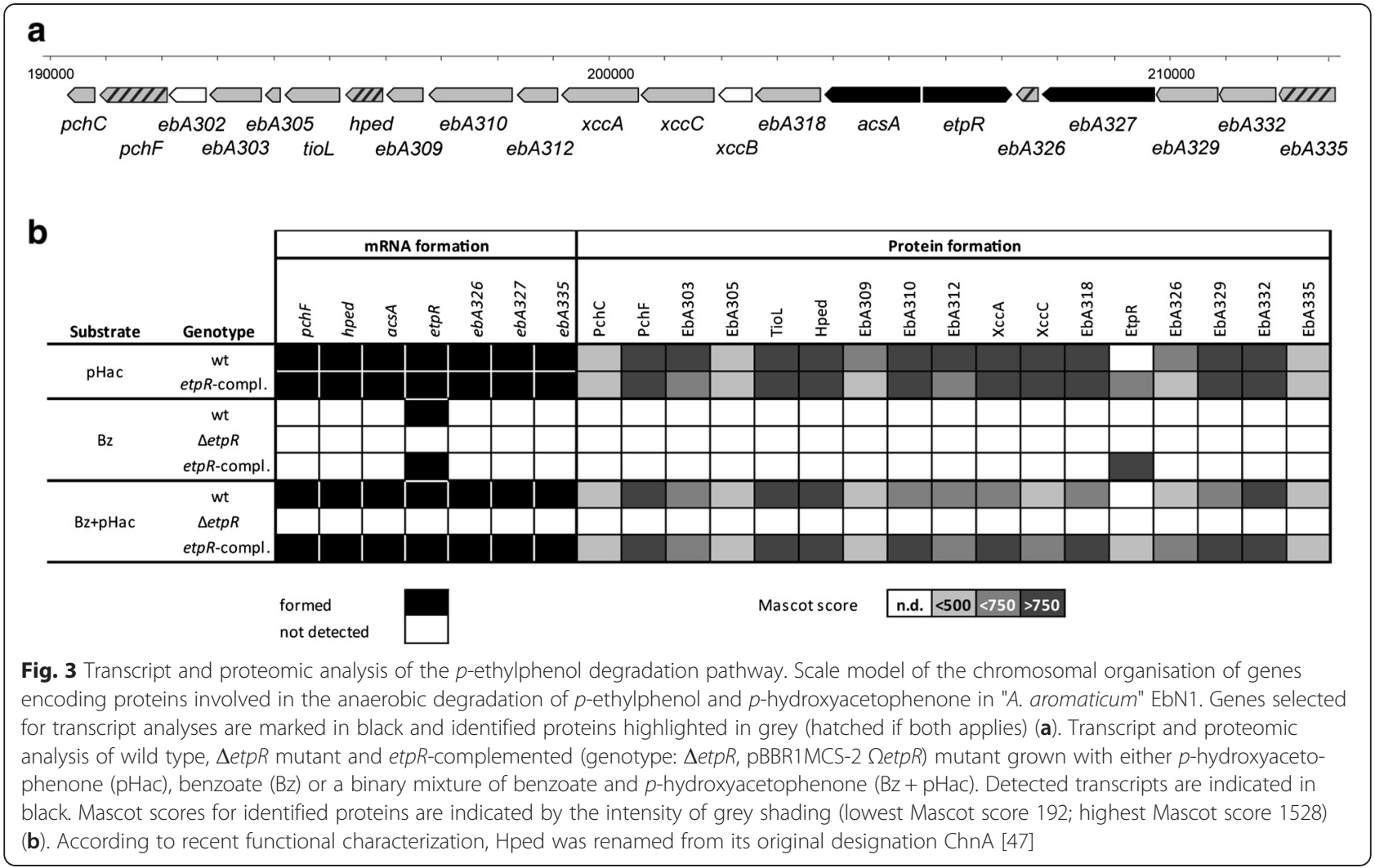

type in presence of benzoate as compared to the $p$ hydroxyacetophenone(effector)-containing conditions. Conversely, a positive feedback may also be unlikely, as the etpR upstream region does not contain a $-12 /-24$ consensus sequence. Taken together, expression of the etpR gene is apparently constitutive.

\section{Wild type EtpR abundance probably sufficient for full- level transcription}

The etpR-complemented mutant strongly expressed etpR under all three tested substrate conditions ( $>28$-fold $\nu s$. wild type; Fig. 4), agreeing with the medium copy number of the vector (around ten), the strong vector-inherent promoter, and the exclusive detection of the EtpR protein. Notably, the artificially high abundance of EtpR in the etpRcomplemented mutant did not yield an increased abundance of proteins involved in $p$-ethylphenol degradation (at $1 / 2 \mathrm{OD}_{\max }$ ) as compared to the wild type (Fig. 3b). Hence, maximum transcription/translation levels are apparently achieved also with the lower EtpR abundance occurring in the wild type, which is also reflected by the similar $\mu_{\max }$ of both strains with $p$-ethylphenol or $p$-hydroxyacetophenone. The higher EtpR level in the etpR-complemented mutant may allow for reaching the maximum levels of catabolic proteins faster, which may explain the significantly shorter lag-phase as compared to the wild type (Fig. 2a, c; Table 1).

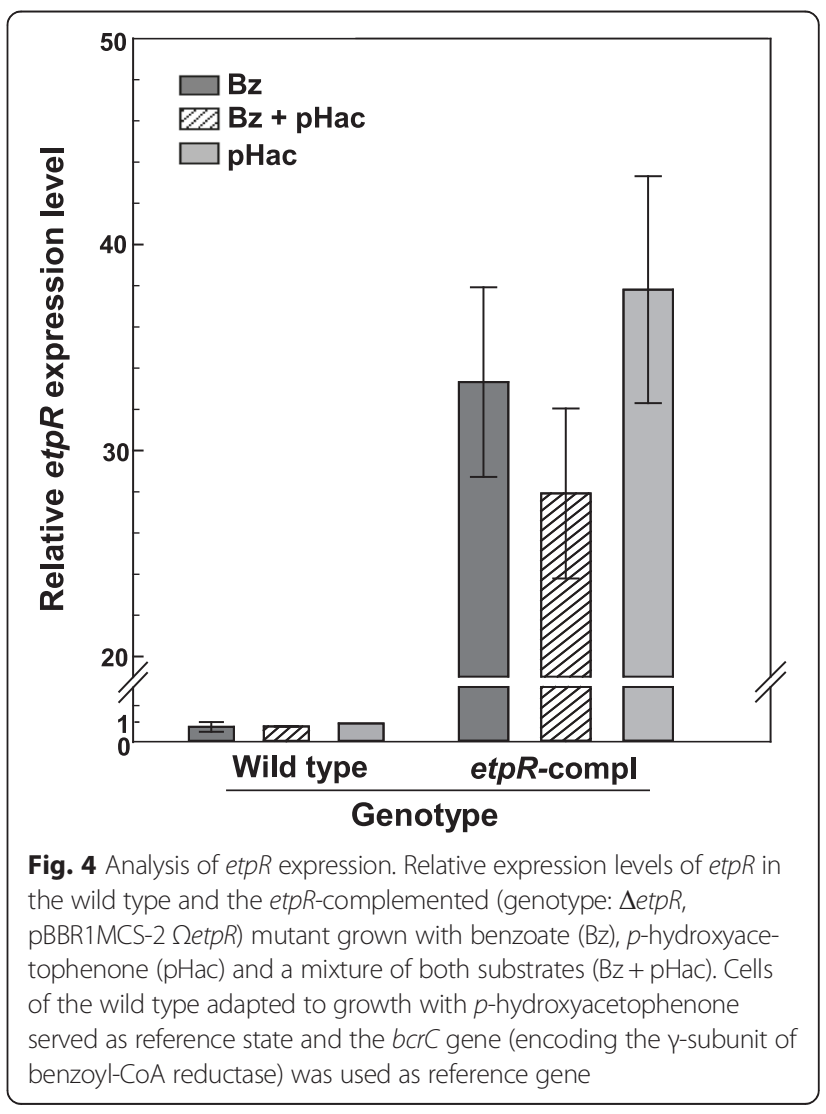




\section{Sequence comparison of regulatory domains}

EtpR belongs to the $\sigma^{54}$-dependent NtrC/XylR-type transcriptional regulators with the typifying architecture of a less well conserved effector-binding $\mathrm{N}$-terminal regulatory domain, connected to the more conserved central activating and C-terminal DNA-binding domains (Fig. 5a) [5, 12, 13].
Known phenol- or alkylbenzene-specific NtrC-type regulators

DmpR and PhlR are closely related regulators of aerobic phenol catabolism in Pseudomonas sp. CF600 [17, 22] and $P$. putida $\mathrm{H}$ [23], respectively. They are activated by direct binding of phenol or derivatives thereof, e.g., cresol and dimethylphenol isomers [24, 25] (Additional file 1: Figure S4). Notably, DmpR also recognizes $o$ - and

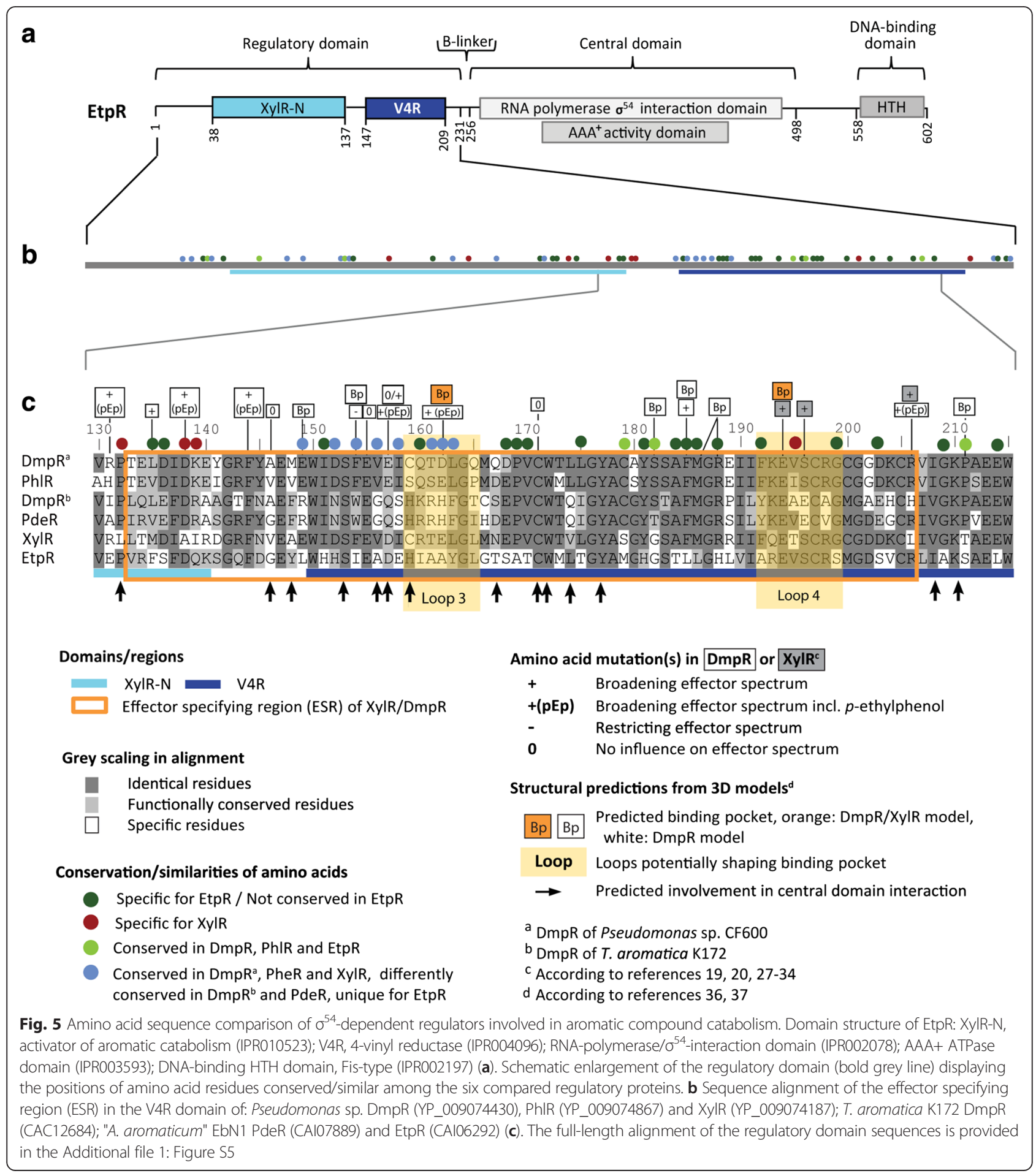


m-ethylphenol [25]. Similarly, DmpR of Thauera aromatica K172 [26] and PdeR of "A. aromaticum" EbN1 $[4,6]$ are suggested to regulate anaerobic phenol degradation; differential proteomics indicated PdeR to also respond to $p$-cresol [4]. XylR of $P$. putida regulates aerobic toluene degradation, and is activated by this as well as other alkylbenzenes such as $m$ - and $p$-xylene [16].

Alteration of regulatory domain residues in XylR and DmpR from Pseudomonas spp. yielded broadened or restricted effector spectra, or even completely inhibited the response to aromatic effectors [19, 20, 27-34]. Most of these residues are confined to a stretch of 75 amino acids, defined as effector specifying region (ESR) [35]. 3D-models of XylR and DmpR predicted structural features for shaping an effector-binding pocket and interaction with the central domain [36, 37]. The current knowledge on the regulatory domain is compiled in Additional file 1: Figure S5.

\section{Comparison of EtpR to known regulators}

The phylogeny of the inspected regulatory domains reflects effector spectra as well as deployment for anaerobic vs. aerobic catabolism. EtpR branches off from the "anaerobic" phenol-specific regulators (PdeR of "A. aromaticum" EbN1 and DmpR of T. aromatica K172), while the three together separate from those of aerobic phenol degradation (DmpR and PhlR of Pseudomonas spp.). The alkylbenzene-specific XylR of P. putida is more distantly related to the other five regulators (Additional file 1 : Figure S4).

Current proteomic data demonstrated specific formation of the " $p$-ethylphenol"-proteins only in cells anaerobically growing with $p$-ethylphenol or $p$-hydroxyacetophenone, but not with 20 other aromatic substrates $[5,11]$. Therefore, activation of EtpR may be restricted to the former two. Such a narrow effector spectrum would distinct EtpR from the above described three regulators of Pseudomonas spp..

To identify primary sequence features, possibly linked to the effector spectrum of EtpR, aligned regulatory domain sequences were analyzed (Additional file 1: Figure S5), with the ESR emphasized in Fig. 5 (amino acid positions 130-205 in EtpR). Across these six complete regulatory domains, 43 residues $(20.7 \%)$ were strictly conserved, comprising most of the reported conserved residues of NtrC-type regulators [36]. Sixty-two other residues (29.8 $\%$; colored circles) are conserved to differing degrees and are mostly ESR-located; they may therefore be effector specifying for EtpR and are briefly described in the following.

Recognition of the phenolic moiety may involve 8 residues (4 in the ESR; brown circles) that are exclusively conserved in the five phenolic compound-sensing regulators (incl. EtpR). Further 6 residues (light green circles) could also contribute to phenol-specificity, as they are conserved in DmpR, PhlR and PdeR, but not in alkylbenzene-sensing XylR and alkylphenol-sensing EtpR.

Notably, a total of 28 residues (dark green circles) are conserved in all proteins except for EtpR, with 16 of them located in the ESR. The sensory relevance of these residues is reflected by four of them being located in the predicted binding pocket of DmpR and XylR [36] and two at locations that broaden the effector spectrum of DmpR [30, 31]. From an inversed perspective, these 28 residues are distinct in EtpR. They may therefore be involved in specific sensing of, e.g., the keto group in $p$ hydroxyacetophenone, since the latter is not known to be an effector of the other five compared regulators. Alternatively, these 28 residues may at least indirectly contribute to a structural shaping of the EtpR-specific sensory properties. These scenarios may also account for another group of 20 residues (blue circles), which are differently conserved in DmpR, XylR and PhlR of Pseudomonas spp. as compared to DmpR of T. aromatica and PdeR of "A. aromaticum" EbN1, but are again distinct in EtpR.

More than 17 residue changes (marked by a "+" sign) in the regulatory domains of Pseudomonas DmpR and XylR (13 in the ESR or close by) broaden their effector spectra (partly also towards $p$-ethylphenol) [19, 31, 34]. They may not be effector specifying for EtpR, as none of the changes yields EtpR-residues.

Notably, the effector specificity of EtpR may not only be attributed to single residues, since also the interaction of different regions contributes to defining effector spectra, i.e., loops (yellow areas) and interactions of regulatory and central domain (black arrows) [36].

\section{Conclusions}

Aerobic aromatic compound degradation pathways of Pseudomonas spp. may accommodate several, structurally related aromatic substrates, e.g., toluene, $m$ - and $p$ xylene as well as 1,2,4-trimethylbenzene in case of $P$. putida [21]. This substrate promiscuity of single pathways is also reflected in rather broad effector spectra of the involved transcriptional regulators $[16,25]$. In contrast, the substrate ranges of the individual anaerobic pathways in "A. aromaticum" EbN1 appear to be more restricted and the substrate-specific expression of their genes is assumed to be individually controlled by corresponding one- or two-component regulatory systems [11]. The observed highly $p$-ethylphenol/ $p$-hydroxyacetophenone-specific induction of the anaerobic $p$-ethylphenol degradation pathway by EtpR can be attributed to a concurrence of unique sets of amino acids accumulating in the ESR (accounting for $36.8 \%$ ), in particular between position 149 and 192. Since they are most 
specific for EtpR as compared to the other regulators, they possibly specify the sensing of phenolic-, ketonicand/or alkylbenzylic-moieties, and determine the observed narrow effector spectrum of EtpR. This further supports a general role of ESRs in mediating sensory recognition of aromatic compounds as previously reported for DmpR and XylR of Pseudomonas spp. [35]. The apparent constitutive, low level expression of etpR in the wild type allows for a full level formation of catabolic proteins that may be only reached faster at higher regulator abundance as observed for the etpR-complemented mutant. Hence, the extent of " $p$-ethylphenol" gene cluster expression should depend on the presence of the substrates/effectors $p$ ethylphenol and $p$-hydroxyacetophenone rather than on the abundance of the regulator itself (EtpR). Overall, the present study demonstrates EtpR (EbA324) to serve as transcriptional regulator in the $p$-ethylphenol catabolism, and represents to our knowledge the first molecular genetic study on a $\sigma^{54}$-dependent regulator in an anaerobic aromatic compound degrader.

\section{Methods}

\section{Bacterial strains and cultivation}

"A. aromaticum" EbN1 and E. coli S17-1 were cultivated as described previously [3,38]. Strains used and generated in this study are summarized in Table 2.

\section{Generation of the in frame $\Delta$ etpR deletion mutation}

Genomic DNA and plasmids were isolated according to standard methods [39]. Oligonucleotide primers were designed using the Lasergene software (version 7.0; DNASTAR, Madison, WI, USA) and purchased from Biomers $\mathrm{GmbH}$ (Ulm, Germany). Nucleotide sequences of applied primers and primer details are provided in Table 3.
For unmarked knockout of the etpR (ebA324) gene, a knockout vector based on the suicide vector pK19mob$s a c B$ [38] was constructed in an E. coli S17-1 background, containing $2.4 \mathrm{kbp}$ of the $5^{\prime}$-region and $1.3 \mathrm{kbp}$ of the 3'-region of etpR. Initially, the 5 '-region containing parts of the acs $A$ gene were cloned into pK19mobsacB using SphI and BamHI restriction sites as reported [38], yielding the plasmid pK19 $\Omega$ acs $A$. Subsequently, the 3'region, containing $e b A 326$ and parts of the $e b A 327$ gene, was cloned into plasmid pK19 $\Omega$ acsA using the BamHI restriction site, resulting in the pK19 $\Omega$ acsAebA326/7 knockout vector (Table 2). In the final vector construct, the start and stop codons of etpR were maintained, separated by a 6 bp BamHI restriction site ("GTGGGATCCACT" blow up in Fig. 1a). Homologous regions were amplified by PCR from genomic DNA of "A. aromaticum" EbN1 using a high fidelity polymerase (Phusion"; ThermoFisher Scientific, Dreieich, Germany; Table 3). The correctness of nucleotide sequences was verified by sequence analysis as described before [38] and pK19 $\Omega$ acsAebA326/7 was transferred by conjugation from the $E$. coli S17-1 donor strain to "A. aromaticum" EbN1 according to the protocol described previously [38]. Integration of the knockout vector (single-cross over) gave rise to kanamycin resistant colonies and was verified by PCR using a primer pair $\left(\Delta e t p R \_F / R\right)$ targeting the up- and downstream intergenic regions of etpR, yielding two amplicons of $318 \mathrm{bp}$ and $2.13 \mathrm{kbp}$, respectively (Table 3; Fig. 1). The second cross-over (i.e., removal of the plasmid) was induced by several transfers of the single cross-over mutant in liquid medium without kanamycin. Colonies capable of growing on sucrosecontaining medium were screened using the same primer pair as described above to identify a $\Delta e t p R$ genotype in

Table 2 Strains and plasmids used in this study

Genotype and/or characteristics

Reference

\begin{tabular}{|c|c|c|}
\hline \multicolumn{3}{|l|}{ Strains } \\
\hline "Aromatoleum aromaticum" EbN1 & Wild type & {$[3]$} \\
\hline EbN1-RR001 ( $\Delta$ etpR mutant) & $\Delta e t p R$ & This study \\
\hline EbN1-RR002 (etpR-complemented mutant) & $\Delta e t p R, \mathrm{pBBR} 1 \mathrm{MCS}-2 \Omega \operatorname{etp} R$ & This study \\
\hline EbN1-RR003 (Wild type containing pBBR1MCS-2) & Wild type, pBBR1MCS-2 & This study \\
\hline EbN1-RR004 ( $\Delta e t p R$ mutant containing pBBR1MCS-2) & $\Delta e t p R, p B B R 1 M C S-2$ & This study \\
\hline Escherichia coli S17-1 & Pro, thi, hsdR, $\operatorname{Tra}^{+}$, recA ${ }^{-}, \operatorname{Tr}^{r}, \mathrm{Sm}^{r}$, $\Omega$ RP4-TE::Mu-Kn::Tn7 & [45] \\
\hline \multicolumn{3}{|l|}{ Plasmids } \\
\hline pK19mobsacB & $\mathrm{Km}^{\mathrm{R}}$, sacB modified from B. subtilis, lacZa & [46] \\
\hline pK19 תacsA & $\begin{array}{l}\mathrm{Km}^{\mathrm{R}} \text {, sacB modified from B. subtilis, lacZa, acsA from } \\
\text { "A. aromaticum" EbN1 }\end{array}$ & This study \\
\hline pK19 ЛacsAebA326/7 & $\begin{array}{l}\mathrm{Km}^{\mathrm{R}} \text {, sacB modified from B. subtilis, lacZa; acsA, ebA326 } \\
\text { and part of ebA327 from "A. aromaticum" EbN1 }\end{array}$ & This study \\
\hline pBBR1MCS-2 & $\mathrm{Km}^{\mathrm{R}}, \mathrm{mob}, \mathrm{lacZa}$ & {$[40]$} \\
\hline pBBR1MCS-2 ЛetpR & $\mathrm{Km}^{R}$, mob, lacZa, etpR from "A. aromaticum" EbN1 & This study \\
\hline
\end{tabular}


Table 3 Oligonucleotide primers applied in this study

\begin{tabular}{|c|c|c|c|}
\hline Primer $^{a}$ & Target gene & Nucleotide sequence $\left(5^{\prime} \rightarrow 3^{\prime}\right)^{\mathrm{b}}$ & Product length $(\mathrm{bp})$ \\
\hline \multicolumn{4}{|c|}{ Gene specific primer pairs } \\
\hline bcrC_108_F & bcrc & CAAGTGGTGGCAACGATGTGT & 191 \\
\hline bcrC_299_R & & GAAGGTCTGGCGATACTGGATGC & \\
\hline pchF_1336_F & pchF & GGCCGGCAACGTCATCATC & 273 \\
\hline pchF_1099_R & & CCATCCGGGAGCACCACT & \\
\hline hped_153_F & hped & TGATCGAAGGCAAGGGCGGAAAAG & 331 \\
\hline hped_473_R & & GCGGCGGTGTAGGGCGTGATG & \\
\hline acsA_1373_F & $\operatorname{acs} A$ & GCCGCGGTGAGGTT & 306 \\
\hline acsA_1068_R & & CGGGGTGAATGTCCA & \\
\hline etpR_425_F & $\operatorname{etp} R$ & AATTGGCCGCTCTTCTG & 310 \\
\hline etpR_116_R & & TTTCGGCATGTTTGTCA & \\
\hline etpR_1717_F $F^{c}$ & etpR & TGGCGACGGCATTCTC & 219 \\
\hline etpR_1499_R $R^{\mathrm{C}}$ & & TGCCGCATCTGTTCACC & \\
\hline ebA326_41_F & ebA326 & TGGCTGGATCTCTGCTC & 275 \\
\hline ebA326_315_R & & TTCCCGTGCGACCTG & \\
\hline ebA327_1070_F & $e b A 327$ & GCTTCGCGGTCCTGA & 375 \\
\hline ebA327_1480_R & & TGTCGCGGTTGTAGC & \\
\hline ebA329_212_F & ebA329 & TGCGGCCCCTGATG & 316 \\
\hline ebA329_537_R & & ACGATGCCGCTGTGG & \\
\hline ebA332_488_F & ebA332 & CCGGCGTGGAGGTAG & 285 \\
\hline ebA332_772_R & & GGCGCGGGGTITT & \\
\hline ebA335_1092_F & ebA335 & GCTGGGGGAGACGAA & 253 \\
\hline ebA335_1344_R & & CGCCGCCTTGTTGT & \\
\hline \multicolumn{4}{|c|}{ Generation of $\Delta e t p R$ deletion mutation } \\
\hline acsA_BamHI_F & $\operatorname{acs} A$ & AAGGATCCCACGAAATGTCTCCTGAACCCTGC & 1300 \\
\hline acsA_Sphl_R & & ACCGGGCATGCGCCCACCAGC & \\
\hline ebA327_BamHI_F & ebA326/ebA327 & GATCAGGATCCACGTCACCG & 2350 \\
\hline ebA326_BamHI_R & & AAGGATCCTGACCGTCGGAGGACCGGATAGATC & \\
\hline \multicolumn{4}{|c|}{ Identification of $\Delta e t p R$ genotype } \\
\hline$\Delta e t p R \_F$ & $3^{\prime}-I_{\mathrm{R}-e t p}{ }^{\mathrm{f}}$ & TGGGCGTAGCGTAGT & $2133^{d} / 318^{e}$ \\
\hline$\Delta e t p R \_R$ & $5^{\prime}-\operatorname{IR}-\operatorname{etp} R^{f}$ & TGGATTGTTCTGTAT & \\
\hline \multicolumn{4}{|c|}{ Generation of in trans complementation of etpR } \\
\hline etpR_Xhol_F & $\operatorname{etp} R$ & AACTCGAGCCCACTCCAAGCGTCGAAACACCGGC & 2436 \\
\hline etpR_Kpnl_R & & AAAGGTACCGCTTCGCTCCGGGAAACCAGTGTGCGC & \\
\hline
\end{tabular}

${ }^{\mathrm{a}} \mathrm{R}=$ reverse primer; $\mathrm{F}=$ forward primer

${ }^{\mathrm{b}}$ Recognition sites for restriction enzymes are marked in bold type

'Primer pair applied in real time RT-PCR experiment

d"A. aromaticum" EbN1 (wild type)

eDeletion mutant (strain EbN1-RR001; $\Delta$ etpR genotype)

$f_{I R}$, intergenic region

the deletion mutant strain EbN1-RR001 (Fig. 1). The genotype was verified by sequencing.

\section{Complementation of etpR in trans into the $\triangle$ etpR deletion} mutant

A complementation vector for in trans expression of etpR was generated in an E. coli S17-1 background, based on the broad-host range vector pBBR1MCS-2 [40]. A $2.4 \mathrm{kbp}$ nucleotide sequence was amplified by PCR, containing $\operatorname{etpR}$ as well as 300 bp upstream of the $\operatorname{etpR}$ start codon to also include the ribosomal binding site (Table 3; Fig. 1). This amplicon was cloned into the pBBR1MCS-2 vector using KpnI and XhoI restriction sites and verified by sequencing. The vector was 
transferred via conjugation to the $\Delta e t p R$ mutant yielding the etpR-complemented mutant strain EbN1-RR002 (genotype: $\triangle e t p R$, pBBR1MCS-2 $\Omega e t p R$ ) (Table 2; Fig. 1). Conjugation via agar-plate mating, identification for positive clones on selective media and PCR-based verification were carried out as previously described [38]. For control experiments, the pBBR1MCS-2 vector without etpR was conjugationally transferred to the wild type strain and the $\triangle e t p R$ mutant yielding strain EbN1-RR003 (genotype: wild type, pBBR1MCS-2) and strain EbN1-RR004 (genotype: $\triangle e t p R, \mathrm{pBBR} 1 \mathrm{MCS}-2$ ), respectively (Table 2 ).

\section{Growth experiments}

Growth experiments with the wild type, the $\Delta e t p R$ mutant and the etpR-complemented mutant were carried out to characterize the phenotype of the generated $\Delta e t p R$ deletion mutation. All three strains were adapted to anaerobic growth with benzoate for at least five passages. Anaerobic cultivation was conducted under nitrate-limited conditions with $400 \mathrm{ml}$ medium in $500 \mathrm{ml}$ flat bottles, sealed with rubber stoppers. Pre-cultures were provided with $4 \mathrm{mM}$ benzoate as growth substrate and cells transferred at half-maximal optical density $\left(1 / 2 \mathrm{OD}_{\max }\right)$ to fresh medium supplemented with either of the following substrates: (i) $p$-ethylphenol $(0.5 \%(\mathrm{w} / \mathrm{v})$ in $14 \mathrm{ml}$ of the inert carrier phase 2,2,4,4,6,8,8-heptamethylnonane (HMN)), (ii) $p$-hydroxyacetophenone $(2 \mathrm{mM})$, (iii) benzoate $(4 \mathrm{mM})$ and (iv) a binary mixture of benzoate $(4 \mathrm{mM})$ and either $p$-hydroxyacetophenone $(2 \mathrm{mM})$ or $p$-ethylphenol $(0.5 \%$ $(\mathrm{w} / \mathrm{v})$ in $10 \mathrm{ml} \mathrm{HMN})$. In case of cultures with benzoate or a binary substrate mixture, the medium contained $10 \mathrm{mM}$ nitrate to achieve higher cell densities; while with $p$-ethylphenol and $p$-hydroxyacetophenone provided as single substrate only $7 \mathrm{mM}$ nitrate were added to the medium.

Growth until depletion of the electron acceptor nitrate was monitored by measuring the optical density at $660 \mathrm{~nm}$ (UV-vis Spectrometer 1240; Shimadzu, Duisburg, Germany) and analysing the substrate concentrations in the culture supernatants with an UltiMate 3000 RSLC system (ThermoFisher Scientific, Germering, Germany). The supernatants were diluted and acidified $(\mathrm{pH} 2.0$, $6 \%$ acetonitrile) prior to analysis. Separation of analytes was achieved with a Dionex Acclaim 120 reversedphase separation column $(250 \mathrm{~mm}$ length, $2.1 \mathrm{~mm}$ inner diameter, $5 \mu \mathrm{m}$ bead size; ThermoFisher Scientific). The column was temperature controlled at $25{ }^{\circ} \mathrm{C}$ and operated with a non-linear gradient of acetonitrile $(5-90 \%(\mathrm{v} / \mathrm{v}), \mathrm{pH} 2.8)$ as eluent at a flow rate of $0.5 \mathrm{ml} \mathrm{min}{ }^{-1}: 2 \mathrm{~min}$ at $5 \%, 5$ to $14 \%$ in $1 \mathrm{~min}, 14$ to $39 \%$ in $10.5 \mathrm{~min}, 39$ to $90 \%$ in $3 \mathrm{~min}$, and $3 \mathrm{~min}$ at $90 \%$. Retention times (compound-specific wavelengths in parentheses) were the following: $p$-ethylphenol, $16.6 \mathrm{~min}$ $(220 \mathrm{~nm}) ; p$-hydroxyacetophenone, $9.4 \mathrm{~min}(270 \mathrm{~nm})$; and benzoate, $11.8 \mathrm{~min}(236 \mathrm{~nm})$.
For selected samples nitrate concentrations were determined by means of an ICS 1100 ion chromatography system (ThermoFisher Scientific). Nitrite and nitrate were separated using an IonPac ${ }^{\mathrm{Tx}}$ AG9-HC separation column (250 mm length, $4 \mathrm{~mm}$ inner diameter, $9 \mu \mathrm{m}$ bead size; ThermoFisher Scientific) and detected at a wavelength of $210 \mathrm{~nm}$. Separation was achieved with sodium carbonate $(9 \mathrm{mM})$ as the eluent administered at a flow rate of $1 \mathrm{ml} \mathrm{min}{ }^{-1}$. The retention times (detection limits in parentheses) were as follows: nitrite, $9.1 \mathrm{~min}$ $(1 \mu \mathrm{M})$; nitrate, $13.3 \mathrm{~min}(1 \mu \mathrm{M})$.

To assess the possibility of a passive depletion of $p$ ethylphenol from the medium, benzoate-adapted cells of the wild type were pre-grown with benzoate and transferred to anoxic medium with $p$-ethylphenol as described before. After $15 \mathrm{~h}$, growth was inhibited by the addition of $50 \mu \mathrm{g} \mathrm{ml}^{-1}$ kanamycin. The concentration of $p$-ethylphenol in the cultures was analyzed by the RSLC system as given above.

\section{Cultivation for transcript and proteomic analysis}

For profiling of compound-specific transcripts and proteome signatures, substrate-adapted wild type, the $\triangle e t p R$ mutant and the etpR-complemented mutant were grown anaerobically with either (i) $p$-hydroxyacetophenone, (ii) benzoate or (iii) a binary mixture of benzoate and $p$-hydroxyacetophenone as sole organic substrate(s). Cells were cultivated in $250 \mathrm{ml}$ flat bottles with $200 \mathrm{ml}$ medium and harvested during linear growth at $1 / 2 \mathrm{OD}_{\max }$ as described by Champion et al. [41]. For each strain and growth condition, three replicate cultures were harvested for transcript and proteomic analyses, respectively (in total 6 replicate cultures each). Cell pellets for transcript analysis were treated with RNAprotect ${ }^{\circ}$ Bacteria Reagent (Qiagen GmbH, Hilden, Germany) according to the manufacturer's instructions.

\section{Preparation of mRNA and reverse transcription (RT)-PCR}

Preparation of total RNA was performed according to the protocol of Oelmüller et al. (22) using cells treated with RNAprotect ${ }^{\bullet}$ Bacteria Reagent. Complete removal of DNA from the RNA preparation was verified by PCR. The quality (i.e., integrity) of the isolated RNA was analysed with MOPS-gels according to standard protocols [39]. cDNA was generated from two independent RNA preparations per strain and substrate condition, respectively, using the antisense primer of the target genes (Table 3). Reverse transcription was performed with $2.5 \mu \mathrm{g}$ RNA applying the RevertAid H Minus Reverse Transcriptase (ThermoFisher Scientific) according to the manufacturer's instructions. cDNA was amplified by PCR using the PCR MasterMix (Promega, Mannheim, Germany). Depending on the PCR efficiency of the gene-specific primer pairs, 1.0 or $2.0 \mu \mathrm{l}$ of cDNA 
preparation were used as template per $20 \mu \mathrm{l} \mathrm{PCR}$ experiment comprising 20 or 39 PCR cycles.

To exclude polar effects resulting from the in frame $\triangle e t p R$ deletion mutation and to qualitatively study gene expression of the two "p-ethylphenol" gene clusters located up and downstream of $\operatorname{etpR}$, transcripts representative of both of them (Fig. 3) were analysed. Target genes were chosen such that both the first and last genes as well as the middle of each of the two gene clusters were covered, i.e., acs $A$, hped and $p c h F$ for the "catabolic" gene cluster, and $e b A 335, e b A 327$ and $e b A 326$ for the "efflux" gene cluster.

\section{Real-time RT-PCR}

Relative expression levels of etpR in the wild type and the eptR-complemented mutant were determined by real-time RT-PCR. BcrC (encoding the catalytic $\gamma$ subunit of benzoyl-CoA reductase) was selected as reference gene since benzoyl-CoA is a common intermediate in anaerobic degradation of benzoate as well as $p$-hydroxyacetophenone, and since $b c r C$ expression is not regulated under the two investigated substrate conditions [5]. cDNA was generated from three individual RNA preparations per strain and growth condition as described above. Real-time PCR was carried out as reported by Kühner et al. [7] using an iQ5 real-time PCR detection system (Bio-Rad, Munich, Germany) and a qPCR MasterMix Plus for SyBR green I with fluorescin (Eurogentec, Cologne, Germany). The correctness of obtained PCR products was verified by sequencing. Determination of PCR efficiencies was performed as described by Ramakers et al. [42] and relative expression levels were calculated according to Pfaffl et al. [43]. At least three replicates of each individual cDNA preparation were analysed (in total 18 qPCR experiments). The wild type grown with $p$-hydroxyacetophenone served as reference state for calculation of relative gene expression levels (Fig. 4).

\section{Proteomic analysis}

Whole cell shotgun analysis of substrate-adapted cells was performed as described recently [44]. Essentially, tryptic peptides were separated by a nanoLC system (UltiMate3000 nanoRSLC; ThermoFisher Scientific) operated in a trap-column mode and equipped with a $25 \mathrm{~cm}$ separation column (C18, $2 \mu \mathrm{m}$ bead size, $75 \mu \mathrm{m}$ inner diameter; ThermoFisher Scientific), applying a 280 min linear acetonitrile gradient. The nanoLC eluent was continuously analyzed by an online-coupled iontrap mass spectrometer (amaZon speed ETD; Bruker Daltonik GmbH, Bremen, Germany) using a captive spray ion source (Bruker Daltonik $\mathrm{GmbH}$ ). Per full scan MS (mass range 400-1400 m/z), $20 \mathrm{MS} / \mathrm{MS}$ spectra of the most intense doubly (or more highly) charged ions were acquired applying subsequent precursor exclusion for $0.2 \mathrm{~min}$. Protein identification was performed using the ProteinScape platform (version 3.1; Bruker Daltonik $\mathrm{GmbH}$ ) on an in-house Mascot server (version 2.3; Matrix Science Ltd, London, UK) based on the genome sequence of "A. aromaticum" EbN1 [6] and applying a target-decoy strategy as described [44]. Search results of the three biological replicates per test state were compiled and only proteins identified by at least 2 peptides were considered.

\section{Additional file}

Additional file 1: Figure S1. Sequence verification of the $\triangle e t p R$ mutant. Figure S2. Anaerobic growth with $p$-ethylphenol. Figure S3. Anaerobic growth with a mixture of benzoate and $p$-ethylphenol. Figure S4.

Phylogenetic relationship of the regulatory domains of $\sigma^{54}$-dependent NtrC-type regulators. Figure S5. Amino acid sequence comparison of $\sigma^{54}$-dependent regulators involved in aromatic compound catabolism. (PDF $1242 \mathrm{~kb}$ )

\section{Competing interests}

The authors declare that they have no competing interests.

\section{Author's contributions}

IB, LW and RR designed the experiments. IB performed the molecular genetics and gene expression experiments. IB, MT and MD performed the physiological experiments and the HPLC anaylses. IB and LW performed the proteomic experiments and analyzed the data. IB, LW and RR prepared the manuscript. The final manuscript was read and approved by all authors.

\section{Acknowledgements}

We are grateful to D. Thies (Bremen) and C. Hinrichs (Oldenburg) for technical assistance. This study was supported by the FOL program of the Carl von Ossietzky University Oldenburg and the Deutsche Forschungsgemeinschaft (GRK 1885 "Molecular basis of sensory biology").

Received: 25 June 2015 Accepted: 15 October 2015

Published online: 02 November 2015

\section{References}

1. Widdel F, Rabus R. Anaerobic biodegradation of saturated and aromatic hydrocarbons. Curr Opin Biotechnol. 2001;12:259-76.

2. Widdel F, Musat F. Diversity and common principles in enzymatic activation of hydrocarbons. In: Timmis KN, editor. Handbook of Hydrocarbon and Lipid Microbiology. Berlin: Springer; 2010. p. 984-1009.

3. Rabus R, Widdel F. Anaerobic degradation of ethylbenzene and other aromatic hydrocarbons by new denitrifying bacteria. Arch Microbiol. 1995:163:96-103.

4. Wöhlbrand L, Kallerhoff B, Lange D, Hufnagel P, Thiermann J, Reinhardt R, et al. Functional proteomic view of metabolic regulation in "Aromatoleum aromaticum" strain EbN1. Proteomics. 2007;7:2222-39.

5. Wöhlbrand L, Wilkes H, Halder T, Rabus R. Anaerobic degradation of p-ethylphenol by "Aromatoleum aromaticum" strain EbN1: pathway, regulation, and involved proteins. J Bacteriol. 2008;190:5699-709.

6. Rabus R, Kube M, Heider J, Beck A, Heitmann K, Widdel F, et al. The genome sequence of an anaerobic aromatic-degrading denitrifying bacterium, strain EbN1. Arch Microbiol. 2005;183:27-36.

7. Kühner S, Wöhlbrand L, Fritz I, Wruck W, Hultschig C, Hufnagel P, et al. Substrate-dependent regulation of anaerobic degradation pathways for toluene and ethylbenzene in a denitrifying bacterium, strain EbN1. J Bacteriol. 2005:187:1493-503.

8. Trautwein K, Wilkes $H$, Rabus R. Proteogenomic evidence for $\beta$-oxidation of plant-derived 3-phenylpropanoids in "Aromatoleum aromaticum" EbN1. Proteomics. 2012;12:1402-13.

9. Trautwein K, Lahme S, Wöhlbrand L, Feenders C, Mangelsdorf K, Harder J, et al. Physiological and proteomic adaptation of "Aromatoleum aromaticum" 
EbN1 to low growth rates in benzoate-limited, anoxic chemostats. J Bacteriol. 2012;194:2165-80.

10. Trautwein K, Kühner S, Wöhlbrand L, Halder T, Kuchta K, Steinbüchel A, et al. Solvent stress response of the denitrifying bacterium "Aromatoleum aromaticum" strain EbN1. Appl Environ Microbiol. 2008;74:2267-74.

11. Rabus R, Trautwein K, Wöhlbrand L. Towards habitat-oriented systems biology of "Aromatoleum aromaticum" EbN1 - Chemical sensing, catabolic network modulation and growth control in anaerobic aromatic compound degradation. Appl Microbiol Biotechnol. 2014;98:1-18.

12. Studholme DJ, Dixon R. Domain architectures of $\sigma^{54}$-dependent transcriptional activators. J Bacteriol. 2003;185:1757-67.

13. Schumacher J, Joly N, Rappas M, Zhang X, Buck M. Structures and organisation of AAA+ enhancer binding proteins in transcriptional activation. J Struct Biol. 2006:156:190-9.

14. Bush M, Dixon R. The role of bacterial enhancer binding proteins as specialized activators of $\sigma^{54}$-dependent transcription. Microbiol Mol Biol Rev. 2012;76:497-529.

15. Helmann JD, Chamberlin MJ. Structure and function of bacterial sigma factors. Annu Rev Biochem. 1988;57:839-72.

16. Abril MA, Michan C, Timmis KN, Ramos JL. Regulator and enzyme specificities of the TOL plasmid-encoded upper pathway for degradation of aromatic hydrocarbons and expansion of the substrate range of the pathway. J Bacteriol. 1989;171:6782-90.

17. Shingler V, Bartilson M, Moore T. Cloning and nucleotide sequence of the gene encoding the positive regulator (DmpR) of the phenol catabolic pathway encoded by pVI150 and identification of DmpR as a member of the NtrC family of transcriptional activators. J Bacteriol. 1993;175:1596-604.

18. Fernández S, de Lorenzo V, Pérez-Martín J. Activation of the transcriptional regulator XyIR of Pseudomonas putida by release of repression between functional domains. Mol Microbiol. 1995;16:205-13.

19. Shingler $\mathrm{V}$, Pavel $\mathrm{H}$. Direct regulation of the ATPase activity of the transcriptional activator DmpR by aromatic compounds. Mol Microbiol. 1995;17:505-13.

20. Delgado A, Salto R, Marqués S, Ramos JL. Single amino acids changes in the signal receptor domain of XyIR resulted in mutants that stimulate transcription in the absence of effectors. J Biol Chem. 1995;270:5144-50.

21. Ramos JL, Marqués S, Timmis KN. Transcriptional control of the Pseudomonas TOL plasmid catabolic operons is achieved through an interplay of host factors and plasmid-encoded regulators. Annu Rev Microbiol. 1997;51:341-73.

22. Powlowski J, Shingler V. Genetics and biochemistry of phenol degradation by Pseudomonas sp. CF600. Biodegradation. 1994;5:219-36.

23. Herrmann H, Müller C, Schmidt I, Mahnke J, Petruschka L, Hahnke K. Localization and organization of phenol degradation genes of Pseudomonas putida strain H. MGG Mol Gen Genet. 1995;247:240-6.

24. Herrmann $\mathrm{H}$, Janke D. Involvement of the plasmid pPGH1 in the phenol degradation of Pseudomonas putida strain H. FEMS Microbiol Lett. 1987:43:133-7.

25. Shingler $V$, Moore T. Sensing of aromatic compounds by the DmpR transcriptional activator of phenol-catabolizing Pseudomonas sp. strain CF600. J Bacteriol. 1994;176:1555-60.

26. Breinig S, Schiltz E, Fuchs G. Genes involved in anaerobic metabolism of phenol in the bacterium Thauera aromatica. J Bacteriol. 2000;182:5849-63.

27. Delgado A, Ramos JL. Genetic evidence for activation of the positive transcriptional regulator Xy1R, a member of the NtrC family of regulators, by effector binding. J Biol Chem. 1994;269:8059-62

28. $\mathrm{Ng} \mathrm{LC}, \mathrm{O}^{\prime}$ Neill E, Shingler V. Genetic evidence for interdomain regulation of the phenol-responsive $\sigma^{54}$-dependent activator DmpR. J Biol Chem. 1996;271:17281-6.

29. Salto R, Delgado A, Michán C, Marqués S, Ramos JL. Modulation of the function of the signal receptor domain of XyIR, a member of a family of prokaryotic enhancer-like positive regulators. J Bacteriol. 1998;180:600-4.

30. Wise AA, Kuske CR. Generation of novel bacterial regulatory proteins that detect priority pollutant phenols. Appl Environ Microbiol. 2000;66:163-9.

31. Sarand I, Skärfstad E, Forsman M, Romantschuk M, Shingler V. Role of the DmpR-mediated regulatory circuit in bacterial biodegradation properties in methylphenol-amended soils. Appl Environ Microbiol. 2001;67:162-71.

32. Garmendia J, Devos D, Valencia A, de Lorenzo V. À la carte transcriptional regulators: unlocking responses of the prokaryotic enhancer-binding protein XyIR to non-natural effectors. Mol Microbiol. 2001;42:47-60.
33. De Las Heras A, de Lorenzo V. Cooperative amino acid changes shift the response of the $\sigma^{54}$-dependent regulator XyIR from natural $m$-xylene towards xenobiotic 2,4-dinitrotoluene. Mol Microbiol. 2011;79:1248-59.

34. Pavel H, Forsman M, Shingler V. An aromatic effector specificity mutant of the transcriptional regulator DmpR overcomes the growth constraints of Pseudomonas sp. strain CF600 on para-substituted methylphenols. J Bacteriol. 1994;176:7550-7.

35. Skärfstad E, O'Neill E, Garmendia J, Shingler V. Identification of an effector specificity subregion within the aromatic-responsive regulators $\mathrm{DmpR}$ and XyIR by DNA shuffling. J Bacteriol. 2000;182:3008-16.

36. Devos D, Garmendia J, de Lorenzo V, Valencia A. Deciphering the action of aromatic effectors on the prokaryotic enhancer-binding protein XyIR: A structural model of its N-terminal domain. Environ Microbiol. 2002:4:29-41.

37. Suresh PS, Kumar R, Kumar A. Three dimensional model for N-terminal A domain of DmpR (2-Dimethylphenol) protein based on secondary structure prediction and fold recognition. In Silico Biol. 2010;10:223-33.

38. Wöhlbrand $L$, Rabus R. Development of a genetic system for the denitrifying bacterium "Aromatoleum aromaticum" strain EbN1. J Mo Microbiol Biotechnol. 2009;17:41-52.

39. Green M, Sambrook J. Molecular Cloning: A Laboratory Manual, vol. 1. 4th ed. Cold Spring Harbor, New York: Cold Spring Harbor Laboratory Press; 2012.

40. Kovach ME, Elzer PH, Hill DS, Robertson GT, Farris MA, Roop RM, et al. Four new derivatives of the broad-host-range cloning vector pBBR1MCS, carrying different antibiotic-resistance cassettes. Gene. 1995;166:175-6.

41. Champion KM, Zengler K, Rabus R. Anaerobic degradation of ethylbenzene and toluene in denitrifying strain EbN1 proceeds via independent substrateinduced pathways. J Mol Microbiol Biotechnol. 1999;1:157-64.

42. Ramakers C, Ruijter JM, Lekanne Deprez RH, Moorman AFM. Assumptionfree analysis of quantitative real-time polymerase chain reaction (PCR) data. Neurosci Lett. 2003;339:62-6.

43. Pfaffl MW. A new mathematical model for relative quantification in real-time RT-PCR. Nucleic Acids Res. 2001;29:2002-7.

44. Zech H, Hensler M, Koßmehl S, Drüppel K, Wöhlbrand L, Trautwein K, et al. Adaptation of Phaeobacter inhibens DSM 17395 to growth with complex nutrients. Proteomics. 2013:13:2851-68.

45. Simon R, Priefer U, Pühler A. A broad host range mobilization system for in vivo genetic engineering: transposon mutagenesis in Gram negative bacteria. Bio/Technology. 1983;1:784-91.

46. Schäfer A, Tauch A, Jäger W, Kalinowski J, Thierbach G, Pühler A. Small mobilizable multi-purpose cloning vectors derived from the Escherichia coli plasmids pK18 and pK19: Selection of defined deletions in the chromosome of Corynebacterium glutamicum. Gene. 1994;145:69-73.

47. Büsing I, Höffken W, Breuer M, Wöhlbrand L, Hauer B, Rabus R. Molecular genetic and crystal structure analysis of 1-(4-hydroxyphenyl)-ethanol dehydrogenase from "Aromatoleum aromaticum" EbN1. J Mol Microbiol Biotechnol. 2015;25:327-39.

\section{Submit your next manuscript to BioMed Central and take full advantage of:}

- Convenient online submission

- Thorough peer review

- No space constraints or color figure charges

- Immediate publication on acceptance

- Inclusion in PubMed, CAS, Scopus and Google Scholar

- Research which is freely available for redistribution 